\title{
Will a Higher Language Proficiency Trigger More False Memories?
}

\author{
Chenxi Zhu ${ }^{1, *}$ \\ ${ }^{1}$ University of Toronto, Department of Psychology, Toronto, Canada, M1C 1A4 \\ *Corresponding author. Email: chenxiaurora.zhu@mail.utoronto.ca
}

\begin{abstract}
False memories, defined as an apparent recollection of an event that did not actually occur, have been extensively studied throughout the past three decades. Although many potential causes or risk factors of false memories have been examined, the role that language proficiency might play in producing false memories received little attention. Having noticed this under-explored topic, this paper intends to investigate the impact that language proficiency has on susceptibility to false memories, and hypothesizes that participants with a higher language proficiency would trigger more false recognition. In this study, 101 native Chinese speakers who learned English as a second language were instructed to remember lists of semantically associated words and phonologically associated words in Chinese and English. Their performance on a subsequent recognition memory test was measured in terms of percentage of true recognition and false recognition. Results demonstrated that no significant differences in the mean proportion of false alarms to critical lures were found between English and Chinese. Also, participants with an intermediate proficiency in English produced the most false memories for English critical lures, followed by the high-proficiency group and low-proficiency group. Besides, participants were more likely to falsely recognize semantically associated critical lures when words were studied and tested in Chinese compared to English, but more likely to falsely recognize phonologically associated critical lures when words were studied and tested in English compared to Chinese. Generally, these results supported the role that language proficiency played in producing false memories. However, contrary to the previous hypothesis, an intermediate language proficiency was suggested to elicit more false memories than either a higher proficiency or a lower one. Based on the findings of this study, the biased memories of people, especially the inaccurate eyewitness testimony and unreliable memories of plaintiffs in the legal settings, could be more easily detected so that the possibility of miscarriages of justice would significantly decrease.
\end{abstract}

Keywords: language proficiency, false memory, DRM paradigm, phonological association, semantic association, bilingualism.

\section{INTRODUCTION}

Defined as a phenomenon in which people remember events that did not happen or recall details of events differently from the way they actually occurred [1], a false memory typically have tremendous real-world implications, especially in the legal settings. Specifically, the biased memories of people were found to result in many adverse consequences in court, for example, serious legal accusations based on inaccurate eyewitness testimony or unreliable childhood memories of plaintiffs $[1,2]$, so this topic has attracted increasing public attention and has engaged researchers' immense interest throughout the past three decades. However, most researchers have appeared to examine the causes of false memories or the features of stimuli influencing the rate of false memories [3], whereas the characteristics of people that may affect the susceptibility to false memories, such as language proficiency, received little attention. Having noticed this under-explored area, this paper aims to investigate the effect that language proficiency has on susceptibility to false memories by utilizing the Deese-Roediger-McDermott (DRM) paradigm [4, 5].

As the most renowned and widely-used false memory paradigm in the laboratory, the DRM paradigm basically consists of two phases: the learning phase and the memory test phase. Participants were usually instructed to study a list of words (e.g. butter, food, eat, 
sandwich, jam, milk, toast, slice) that were all semantically associated to a non-presented critical lure (e.g. bread) during the learning phase, while the memory test phase typically involved the recall and recognition of either presented or non-presented words. As previous research indicated, a significant percentage of participants tended to falsely recall or recognize the critical lures, even though they were not presented over the learning phase [5].

Relevant laboratory research incipiently examined the effect of semantic association on false memory among adult participants but subsequently started to focus on children's differential tendency to experience false memories. A robust finding was that not only did adults fall prey to the DRM illusion, but also children were susceptible to false recognition and recall when completing the DRM task $[3,6]$. Besides, children's false recognition and recall for non-presented critical lures were found to increase with age [3, 6]. A reasonable explanation for this phenomenon was that young children with a relatively low proficiency in their native languages usually had less extensive semantic networks than older children and adults, and therefore were less likely to activate the critical lures that were semantically associated to presented words [6]. Based on this explanation, language proficiency was assumed to play a role in the production of false memories.

The majority of relevant research evaluated the effect of semantic association on false memories, whereas false memories for phonologically associated words has not been extensively studied, though this topic was also worthy of attention. Thus, the present study incorporated phonologically associated words into the DRM lists, aiming to examine the false recognition of both semantically associated words and phonologically associated words.

Given the fact that all the recruited participants in the present study were native Chinese speakers who learned English as a second language, three main hypotheses have been suggested. Firstly, with a higher proficiency in Chinese and more readily-activated Chinese semantic networks and phonological networks, participants were expected to produce more false memories when encoding and recognizing words in Chinese rather than English. Secondly, as the performance on language tests was considered as a manifestation of language proficiency, it is reasonable that participants with a higher grade on English exams were more susceptible to false memories for critical words studied and tested in English than those with a relatively lower grade. Finally, since a high language proficiency was positively correlated with the proportion of semantic recognition errors but negatively correlated with the proportion of phonological recognition errors [7], participants were assumed to be more likely to falsely recognize semantically associated critical lures when words were studied and tested in Chinese, but more likely to falsely recognize phonologically associated critical lures when words were studied and tested in English.

\section{METHOD}

\subsection{Participants}

A total of 101 high school students from Handan No.1 High School in China voluntarily participated in this study. The mean age of this group of participants (55 females, 46 males) was 15.57 years $(\mathrm{SD}=0.63$; range $=14-17)$. All of them were fluent, native Chinese speakers and readers who learned English as a second language. On average, participants rated their proficiency in Chinese as $7.79(\mathrm{SD}=1.37)$ on a scale ranging from 1 (beginner) to 10 (native speaker), while they rated their proficiency in English as 5.73 (SD = 1.58 ) on the same scale. They were currently studying English as a subject at high school, but barely spoke English outside of English courses and examinations. All participants understood the precise information of this research and gave their informed consents to participate in this study.

\subsection{Materials}

Participants were presented with 128 -words lists (can also referred to as themes), half of which was semantically related to a critical lure (e.g. DRM list: nurse, sick, health, medicine, hospital, ill, patient, dentist, critical lure: doctor) and the other half was phonologically related to a critical lure (e.g. DRM list: sky, side, cite, sigh, sight, sign, size, slight, critical lure: site). Both semantic and phonological DRM lists were comprised of three themes in Chinese and three themes in English. Specifically, semantic lists in English were selected from the lists employed by Roediger and McDermott [5], while Chinese semantic themes were literally translated from the original English DRM lists. Moreover, phonological lists in English were constructed by adjusting the lists organized by Kreuz as well as Terrell and Meadows [8, 9], whereas Chinese phonological lists were modified from the lists utilized in earlier experiments $[10,11]$. Besides, all of the English DRM lists were additionally adapted to participants' English proficiency and only included words containing nine letters or fewer.

\subsection{Procedures}

Participants were tested individually in a room with a computer monitor at their high school. This study was carried out during two phases: the learning phase and the memory test phase. In the first phase, participants were instructed to remember the words shown on the computer monitor as many as possible in preparation for 
a subsequent memory test. Words were visually displayed on the computer monitor for $2000 \mathrm{~ms}$, with one word presented at a time. The learning phase consisted of three Chinese semantic themes, three English semantic themes, three Chinese phonological themes, and three English phonological themes in total. Both the order of studying semantic and phonological themes and the order of studying themes in Chinese and English was randomly intermixed. In the second phase, participants were required to complete an old-new recognition memory test. This memory test phase was composed of 72 words, including the 12 critical lures, 36 studied words, and 24 unrelated distractors. Participants were asked to press " $\mathrm{f}$ " if they recognized the word as presented before but press " $\mathrm{j}$ " if they experienced the word as new. Both true recognition and false recognition were scored and expressed in percentages. Moreover, participants' proficiency in English was operationalized by the average of midterm marks and final exam marks which were obtained with their permission. Based on their performance on English tests, participants were divided into three groups: the high-proficiency group, the medium-proficiency group, and the low-proficiency group. Besides, digit span was also measured through an additional memory task.

\section{RESULTS}

\subsection{Language type effect on true and false recognition}

Three repeated-measures t-tests were conducted to assess the effect of language type (Chinese vs. English) on the mean percentage of true recognition, false recognition of critical lures, and false recognition of unrelated distractors. As the results indicated in Table 1, participants had higher true recognition in Chinese $(82.92 \%)$ than in English $(81.14 \%)(p<0.05)$. However, regarding false memories in these two conditions, neither critical lures $(39.40 \%$ vs. $35.44 \%)$ nor unrelated distractors (7.94\% vs. 5.96\%) differ between English and Chinese, $t(100)=1.574, p=0.119,95 \%$ CI $[-1.03$, $8.95] ; t(100)=1.934, p=0.056,95 \%$ CI $[-0.05,4.01]$, respectively.

Table 1. Mean percentage of true and false recognition in Chinese and English

\begin{tabular}{|l|l|l|}
\hline & Chinese & English \\
\hline True recognition & $82.92(9.72)$ & $81.14(10.32)$ \\
\hline False recognition of unrelated distractors & $5.96(12.56)$ & $7.94(14.59)$ \\
\hline False recognition of critical lures & $35.44(19.86)$ & $39.40(24.20)$ \\
\hline
\end{tabular}

Note: Standard deviations are reported in parenthesis.

\subsection{Language proficiency effect on true and false recognition in English}

Three one-way between-subjects analyses of variance (ANOVA) were carried out to investigate the impact that proficiency in English (high-proficiency group vs. medium-proficiency group vs. low-proficiency group) had on the mean proportion of true recognition of English words, false recognition of English critical lures, and false recognition of English unrelated distractors. The analysis comparing the mean percentage of false memories for critical lures in English revealed a significant difference among the language proficiency, $F(2,98)=3.246, p<0.05$. As shown in Table 2, the results demonstrated that the medium-proficiency group (47.97\%) produced more false memories for English critical lures than high-proficiency group and low-proficiency group (36.05\% and $34.59 \%$, respectively, all $p \mathrm{~s}<0.05)$, but there was not a significant difference in susceptibility to false recognition of English critical lures between high-proficiency group and low-proficiency group, $p=$ $0.801,95 \%$ CI $[-10.02,12.93]$. The other two analyses regarding the mean proprotion of true recognition of English words and false recognition of English unrelated distractors did not show significant differences, $F(2,98)=1.265, p=0.287 ; F(2,98)=1.920, p=0.152$, respectively. No significant differences in digit span were found between groups, thus the differential percentages of false recognition could not be explained by their differential ability to remember.

Table 2. Mean percentage of false recognition of critical lures in English

\begin{tabular}{|c|c|c|c|c|c|}
\hline & \multirow[t]{2}{*}{$\mathrm{N}$} & \multirow{2}{*}{$\begin{array}{l}\text { Mean } \\
\text { Percentage }\end{array}$} & \multirow{2}{*}{$\begin{array}{l}\text { Standard } \\
\text { Deviation }\end{array}$} & \multicolumn{2}{|c|}{ 95\% Confidence Interval } \\
\hline & & & & Lower End & Upper End \\
\hline High-proficiency group & 30 & 36.05 & 20.21 & 28.50 & 43.60 \\
\hline Medium-proficiency group & 33 & 47.97 & 26.36 & 38.62 & 57.32 \\
\hline Low-proficiency group & 38 & 34.59 & 23.73 & 26.79 & 42.39 \\
\hline
\end{tabular}




\subsection{Language type effect on false recognition of two different types of critical lures}

In order to determine whether language proficiency influences the rate of false memories for two different types of critical lures, a 2 (Type of Language: Chinese vs. English) $\times 2$ (Type of Critical Lures: semantically associated vs. phonologically associated) repeated-measures ANOVA was performed on the percentage of false alarms given to each Type of Language. This ANOVA yielded a significant main effect of Type of Critical Lures, $F(1,100)=4.825, p<$ $0.05, \eta_{\mathrm{p}}^{2}=0.046$, and a significant Type of Language $\times$ Type of Critical Lures interaction, $F(1,100)=57.632$, $p<0.001, \eta_{\mathrm{p}}^{2}=0.366$. The precise data were presented in Table 3. As the main effect of Type of Critical Lures indicated, false alarms to semantically associated critical lures $(\mathrm{M}=40.42, \mathrm{SD}=2.42)$ was higher than false memories for phonologically associated critical lures $(\mathrm{M}=34.42, \mathrm{SD}=2.11)$. Regarding the interaction between Type of Language and Type of Critical Lures, the analyses showed that participants falsely recognized more semantically associated critical lures in Chinese (48.51\%) rather than in English (32.32\%), but falsely recognized more phonologically associated critical lures in English (46.48\%) rather than in Chinese (22.36\%).

Table 3. Mean percentage of false recognition of semantically-associated and phonologically associated critical lures in Chinese and English

\begin{tabular}{|l|l|l|l|}
\hline Percentage of false recognition & Chinese & English & Total \\
\hline Semantically associated critical lures & $48.51(2.89)$ & $32.32(3.17)$ & $40.42(2.42)$ \\
\hline Phonologically associated critical lures & $22.36(2.53)$ & $46.48(3.02)$ & $34.42(2.11)$ \\
\hline
\end{tabular}

Note: Standard deviations are reported in parenthesis.

\section{DISSCUSSION}

Three key results were produced through this experimental research. Firstly, inconsistent with the previous expectation, the results indicated that there was not a significant difference in the mean proportion of false alarms to critical lures between English and Chinese. Secondly, participants with an intermediate proficiency in English produced higher rates of false recognition of critical lures than those who were more proficient or less proficient in this language, which did not support the author's hypothesis as well. Finally, participants were more susceptible to false recognition of semantically associated critical lures while encoding and recognizing words in Chinese rather than English, but were more susceptible to false recognition of phonologically associated critical lures when words were studied and tested in English compared to Chinese. Taken together, the evidence lent support to the assumption that language proficiency played a crucial role in producing false memories in the DRM paradigm. However, the initial prediction that false memory would increase with language proficiency should be abandoned, and a modification should be made to this hypothesis to correspond with the findings: individuals with an intermediate-level language proficiency would produce more false memories than those who were either more proficient or less proficient.

All of the three key results could be explained by this newly-modified hypothesis. Firstly, given the assumption that people with a medium-level language proficiency would produce the most false recognition, the correlation between language proficiency and susceptibility to false memories could be described as an inverted U-shaped relationship. Therefore, if participants' mean proficiency in Chinese was located higher than the intermediate level of language proficiency while their average proficiency in English was located lower than it, it would be possible that participants demonstrated similar percentages of false alarms to critical lures in Chinese and English. Secondly, based on this hypothesis, it would be reasonable that the medium-proficiency group had a higher percentage of false recognition of critical lures than the high-proficiency group and low-proficiency group. Finally, participants were confirmed to make more semantic recognition errors in their native language but more phonological recognition errors in their second language, but this evidence did not contradict the newly-modified hypothesis.

Besides, this experiment ruled out two other possible explanations by instructing participants to finish two additional tasks. Firstly, in order to ensure that the differential percentage of false recognition was caused by differential language proficiency rather than participants' differential ability to remember, the current study required participants to complete an extra memory test that measured their digit span. As the result indicated, there was not a significant difference in digit span between groups. Secondly, participants were also asked to rate whether they were familiar with the words studied and tested in this experiment, so the possibility that being tested on unfamiliar words influenced the rate of false memories could be eliminated. 


\section{CONCLUSION}

In order to examine whether language proficiency played a role in producing false memories, the current experiment utilized the DRM paradigm to measure 101 Chinese high school students' susceptibility to false recognition. As the results indicated, no significant differences in mean percentage of false recognition of critical lures were found between Chinese and English. Results also demonstrated that participants with a medium proficiency in English produced more false memories for English critical lures than the high-proficiency group and low-proficiency group. Also, participants were more susceptible to semantic recognition errors when words were studied and tested in Chinese compared to English, but were more susceptible to phonological recognition errors when words were studied and tested in English compared to Chinese. Given the outcomes of this experiment, it could be concluded that language proficiency had an impact on susceptibility to false memories, and that individuals with an intermediate language proficiency would produce more false memories than those with either a higher proficiency or a lower one.

Based on the findings of this study, the biased memories of people, especially the inaccurate eyewitness testimony and unreliable memories of plaintiffs in the legal settings, could be more easily detected so that the possibility of miscarriages of justice would significantly decrease. In addition to this real-world implication, this study has four main theoretical implications. Firstly, most researchers have focused on the causes of false memories or the features of stimuli influencing the rate of false memories [3], while few experiments were conducted to investigate the characteristics of people that may affect the susceptibility to false memories, such as language proficiency. Thus, the present study aimed to examine this area that received little attention from researchers. Secondly, false memories have been studied in monolingual speakers of many languages, but few research recruited bilingual speakers as participants like the current study did. Thirdly, most relevant research utilized the DRM paradigm to evaluate the effect that semantic association had on false memories, whereas phonological recognition errors and phonological recall errors were largely neglected. Therefore, the present study intended to investigate the false recognition of both semantically associated words and phonologically associated words by incorporating phonologically associated words into the DRM word lists. Finally, laboratory studies regarding false memories initially focused on adults but recently has focused on children's susceptibility to false memories, but few experiments recruited adolescents as participants as the current research did.

\section{AUTHORS' CONTRIBUTIONS} Zhu.

This paper is independently completed by Chenxi

\section{ACKNOWLEDGMENTS}

I would like to thank Denes Szucs for his generous support, Xiaoqian Zhou for her academic suggestions, and Cuihong Wang for her helpful comments on the manuscript.

\section{REFERENCES}

[1] Mark Krause, Daniel Corts, Stephen Smith, \& Dan Dolderman, An Introduction to Psychological Science (2nd ed.), Pearson Education, 2017(3).

[2] Randy J. Larsen, David M. Buss, David B. King, \& Carolyn E. Ensley, Personality Psychology: Domains of Knowledge About Human Nature, McGraw-Hill Education, 2017(12)

[3] Richard L. Metzger, Amye R. Warren, Jill T. Shelton, Jodi Price, Andrea W. Reed, \& Danny Williams, Do Children "DRM" Like Adults? False Memory Production in Children. Developmental Psychology, 2008(1). pp.169-181.

[4] James Deese, On the Prediction of Occurrence of Particular Verbal Intrusions in Immediate Recall. Journal of Experimental Psychology, 1959(7). pp.17-22.

[5] Henry L. Roediger \& Kathleen B. McDermott, Creating False Memories: Remembering Words Not Presented in Lists. Journal of Experimental Psychology: Learning, Memory, and Cognition, 1995(7). pp.803-814.

[6] Henry Otgaar \& Ingrid Candel, Children's False Memories: Different False Memory Paradigms Reveal Different Results. Psychology, Crime \& Law, 2011(7). pp.513-528.

[7] Grant H. Henning, Remembering Foreign Language Vocabulary: Acoustic and Semantic Parameters. Language Learning, 1973(12). pp.185-196.

[8] Roger J. Kreuz, The Subjective Familiarity of English Homophones. Memory \& Cognition, 1987(3). pp.154-168.

[9] C. D. Terrell \& B. Meadows, A List of English Homophones. The Quarterly Journal of Experimental Psychology A: Human Experimental Psychology, 1985(11). pp.627-631.

[10] Yi-Chen Lin, Hsiang-Yu Chen, Yvonne C. Lai, \& Denise H. Wu, Phonological Similarity and Orthographic Similarity Affect Probed Serial 
Recall of Chinese Characters. Memory \& Cognition, 2015(4). pp.538-554.

[11] Wai F. Yik, The Effect of Visual and Acoustic Similarity on Short-Term Memory for Chinese Words. The Quarterly Journal of Experimental Psychology, 1978(8). pp.487-494. 